\title{
Tracing Dissolved Trace Elements from Source to Sink: Spatio- temporal Variability and Baseline Concentrations in Ganges River to Judge Future Changes
}

\author{
SOUMITA BORAL ${ }^{1} \&$ INDRA SEKHAR SEN ${ }^{1}$
}

${ }^{1}$ IIT Kanpur, India; soumita@iitk.ac.in

River water quality is constantly changing because of the increased human population and their associated pollution. For example, alarms have been raised about the increased load of heavy metals and nutrients in many rivers worldwide. The concerns are also valid for the Ganges River in the Indian subcontinent, which drains $980 \times 103 \mathrm{~km} 2$ of land in India and Bangladesh and provide water resource to nearly half a billion people. The physicochemical and biological parameters such as $\mathrm{pH}$, electrical conductivity, dissolved oxygen, biochemical oxygen demand, fecal coliform, and total coliform are well monitored in the Ganges River. There is also a significant body of published information on dissolved trace elements concentrations. However, these studies have only focused in and around large cities and industrial zones to capture the "hotspots" of river contamination. Also, these studies have followed different sampling strategies and analytical protocols, and therefore their direct comparison will produce large discrepancies in results. The existing literature data therefore only gives us a unique snapshot to identify local "hotspots" of trace element pollution and makes our understanding of trace element river geochemistry significantly biased towards geographically smaller areas. It is noteworthy that trace element data that represent the entire drainage of Ganges River is currently missing, and therefore we have limited understanding of how the Ganges river geochemically evolves from source to sink vis-à-vis seasonal and temporal dynamics. We also do not have a baseline to quantify the level of perturbation of the natural state of the river due to human interventions. Here, we present the composition of 17 trace elements from 38 locations across the entire Ganges basin that is spanning from the glacial streams in the Himalayas to downstream, across three seasons based on the Indian Summer Monsoon (ISM): pre-ISM (April-June), ISM (July-September), and post-ISM (October-December) over three years (2014-2016). Nearly 4000 trace element data points were analyzed using statistical, chemometric, and geochemical techniques to elucidate the origin and concentration variability in space and time. Using a combination of a low percentile of the data distribution and average concentrations of the headwater we attempted to determine a baseline concentration of trace elements to judge future changes in the Ganges Basin. 